INPLASY

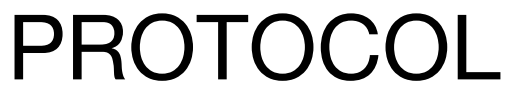

To cite: Yu et al. Meta-analysis of arbidol versuslopinavir/ ritonavir in the treatment of coronavirus disease 2019. Inplasy protocol 202190063. doi:

10.37766/inplasy2021.9.0063

Received: 20 September 2021

Published: 20 September 2021

Corresponding author:

Dengchao Wang

wangdengchaopwk@163.com

Author Affiliation:

Department of General

Surgery, Zigong Fourth

People's Hospital.

Support: None.

Review Stage at time of this submission: Completed but not published.

Conflicts of interest:

None declared.

\section{Meta-analysis of arbidol versuslopinavir/ritonavir in the treatment of coronavirus disease 2019}

Yu, M1; Wang, DC2; Li, S3; Wei, J4; Lei, YH5\% Huang, LY6.

Review question / Objective: It is still controversial whether the effectiveness of Abidol's antiviral treatment for COVID-19 is better than other antiviral treatments. Therefore, timely and systematic evaluation of the therapeutic effects of the Arbidol and lopinavir/ritonavir (LPV/r) on coronavirus disease 2019 is of great significance.

Condition being studied: Coronavirus disease 2019.

Information sources: China Knowledge Network Database (CNKI), WanFang Database (WanFang database), VIP Chinese Science and Technology Journal Database (VIP), PubMed, Embase, and Cochrane Library.

INPLASY registration number: This protocol was registered with the International Platform of Registered Systematic Review and Meta-Analysis Protocols (INPLASY) on 20 September 2021 and was last updated on 20 September 2021 (registration number INPLASY202190063).

\section{INTRODUCTION}

Review question / Objective: It is still controversial whether the effectiveness of Abidol's antiviral treatment for COVID-19 is better than other antiviral treatments. Therefore, timely and systematic evaluation of the therapeutic effects of the Arbidol and lopinavir/ritonavir (LPV/r) on coronavirus disease 2019 is of great significance.

Condition being studied: Coronavirus disease 2019. 


\section{METHODS}

Participant or population: Patients with COVID-19advanced rectal cancer.

Intervention: Arbidol.

Comparator: lopinavir/ritonavir.

Study designs to be included: Randomized controlled trials, cohort studies, retrospective controlled trials.

Eligibility criteria: (1) Patients with confirmed coronavirus disease 2019; (2) Age $\geq 18$ years old; (3) Hospital stay longer than 14 days;(4) Intervention measures: use of Arbidol or lopinavir/ritonavir to treat coronavirus disease 2019 and compare the effects of the two groups.

Information sources: China Knowledge Network Database (CNKI), WanFang Database (WanFang database), VIP Chinese Science and Technology Journal Database (VIP), PubMed, Embase, and Cochrane Library.

Main outcome(s): Rate of positive-tonegative conversion of SARS-CoV-2 nucleic acid on Day 7, rate of positive-to-negative conversion of SARS-CoV-2 nucleic acid on Day 14, rate of improvement on chest CT on Day 14, rate of cough disappearance on Day 14, rate of progression to severe/ critically illness, rate of mortality and incidence of adverse reactions.

Quality assessment / Risk of bias analysis: The randomized trial used the modified Jadad et al. scoring scale to evaluate the quality of the included studies from the following four aspects: (1) whether the random method was used (appropriate 2 points, unclear 1 point, inappropriate 0 points); 2) whether there was allocation hiding ( 2 points for proper, 1 point for unclear, 0 points for inappropriate or unused); (3) whether a blinding method was used ( 2 points for appropriate, 1 point for unclear, 0 points for inappropriate); and (4) whether there was loss to follow-up or withdrawal ( 1 point if described, 0 points if not described). Four were case-control studies, so the Newcastle-Ottawa Scale (NOS) was used for quality evaluation. According to the scoring system of NOS scale, we checked the selection (4 points), comparability ( 2 points) and outcome/ exposure (3 points) of each study. Studies with a score of $>7$ were considered to have a low risk of bias, studies with a score of 5-7 had a moderate risk of bias, and studies with a score of $<5$ had a high risk of bias. Exclude articles with a high risk of bias. For randomized clinical trials, in addition to using the modified Jadad scoring scale, we also use the Cochrane collaborative tool to assess the risk of bias.

Strategy of data synthesis: RevMan $\mathbf{5 . 3}$ provided by the Cochrane Collaboration was used for data analysis.The rate of positive-to-negative conversion of SARSCoV-2 nucleic acid on day 7 , rate of positive-to-negative conversion of SARSCoV-2 nucleic acid on day 14 , rate of cough disappearance on day 14 , rate of improvement of chest CT on day 14, rate of becoming severely/critically ill, rate of mortality and incidence of adverse reactions as binary variables, OR(odds ratio) and its $95 \% \mathrm{CI}$ (confidence interval, CI) were used as the effect value.For continuous variables, MD and its $95 \% \mathrm{CI}$ (confidence interval, $\mathrm{Cl}$ ) were used as the effect value. If there was no significant difference by the $Q$ test $(P>0.10,12 \leq 50 \%)$, the fixed-effects model was used for the meta-analysis.

Subgroup analysis: If there was a significant difference ( $P \leq 0.10$ and $12>50 \%$ ), sensitivity analysis or subgroup analysis was conducted to find out the source of heterogeneity.

Sensitivity analysis: If there was a significant difference ( $P \leq 0.10$ and $I 2>50 \%$ ), sensitivity analysis or subgroup analysis was conducted to find out the source of heterogeneity, and the source of heterogeneity was eliminated check whether the results are the same. If the heterogeneity test cannot be carried out and the source of heterogeneity cannot be eliminated, it is considered that the statistical heterogeneity between studies is 
too large for comparative analysis, so only descriptive analysis is carried out.

Country(ies) involved: China.

Keywords: coronavirus disease 2019;

Arbidol; lopinavir/ritonavir; meta-analysis.

Contributions of each author:

Author 1 - Miao Yu.

Author 2 - Deng-Chao Wang.

Author 3 - Sheng Li.

Author 4 - Yue-Hua Lei.

Author 5 - Wei Jian.

Author 6 - Li-Yan Huang. 